Michael Hüther, Markos Jung

\title{
Unzureichende Investitionsoffensive
}

Die Corona-Krise hat den Staat in seiner elementaren Schutzfunktion in Zeiten großer Not und Bedrohung herausgefordert. Das hat inm aus der Sicht vieler eine neue Bedeutung verschafft. Infolge der medizinischen, wirtschaftlichen und sozialpolitischen Krisenreaktionen stieg die deutsche Staatsquote 2020 auf über $51 \%$ des Bruttoinlandsprodukt (BIP) an - von $45 \%$ in der vergangenen Dekade. Neben diesen kurzfristigen Maßnahmen sollen mittelfristig angelegte Wachstumsprogramme wie der EU-Wiederaufbaufonds oder starke Erhöhungen der Investitionsausgaben des Bundes dazu beitragen, aus der Krise herauszuwachsen. Aus der Wirtschaftsgeschichte kennen wir das Phänomen des „Niveauverschiebungseffekts" (Displacement-Effekt nach Peacock und Wiseman, 1961), wonach die Ausgabenquote des Staates nach einem exogenen Schock - wie Krieg oder Naturkatastrophe - nicht mehr auf das Vorkrisenniveau zurückfällt. Es ist in der Tat zu vermuten, dass die in der Pandemie aufgedeckten grundsätzlichen strukturellen Probleme längerfristig Folgen haben werden, zumal es sowohl auf nationaler als auch auf europäischer Ebene vor allem um Investitionslücken geht, die zu schließen sind.

\section{Beachtliche öffentliche Investitionslücke}

Die Erhöhung öffentlicher Investitionen ist jedoch nicht nur aus krisenbedingter Sicht geboten. Vielmehr bestanden bereits vor 2020 beachtliche Investitionsbedarfe (Bardt et al., 2019). Diese ergeben sich zum einen aus einer Reihe gesamtgesellschaftlicher transformatorischer Aufgaben (Dekarbonisierung, digitale Transformation, demografischer Wandel). Zum anderen blicken wir auf eine lange Phase un-

(C) Der/die Autor:in(nen) 2021. Open Access: Dieser Artikel wird unter der Creative Commons Namensnennung 4.0 International Lizenz veröffentlicht (creativecommons.org/licenses/by/4.0/deed.de).

Open Access wird durch die ZBW - Leibniz-Informationszentrum Wirtschaft gefördert.

Prof. Dr. Michael Hüther ist Direktor des IW in Köln.

Dr. Markos Jung ist persönlicher Referent des IWDirektors in Köln. zureichender öffentlicher Investitionstätigkeit zurück. Zwar lag das preisbereinigte Bruttoanlagevermögen des Staates im Jahr 2019 rund 14\% höher als zur Jahrtausendwende - bei Bund und Ländern waren es $23 \%$ respektive $30 \%$. Die These, der Staat als Ganzes verzehre seinen Kapitalstock kann damit zunächst entkräftet werden (Grömling et al., 2019b). Allerdings lag das preisbereinigte Bruttoanlagevermögen der Gemeinden - immerhin Hauptträger der öffentlichen Investitionen - 2019 nur 5\% höher als 2000, im Zehnjahresvergleich ist es überhaupt nicht gewachsen. Zur Identifizierung einer möglichen Investitionslücke genügt allerdings der reine Blick auf die Entwicklung staatlicher Investitionsausgaben und Anlagevermögen nicht. So beschreibt eine isolierte Betrachtung der Entwicklung von Kapitalstock oder Investitionen nur die rein pekuniäre Dimension öffentlicher Investitionstätigkeit, sagt aber noch nichts darüber aus, ob dieser Zuwachs insgesamt ausreichend und im Detail angemessen ist, ob staatliche Ausgaben effizient verwendet werden und effektiv nutzbar sind oder wie hoch ein volkswirtschaftlich optimales Niveau öffentlichen Kapitals wäre (Feld et al., 2020; Grömling et al., 2019a).

Einen möglichen Bewertungsansatz stellt der Vergleich der Kapitalstockentwicklung mit anderen volkswirtschaftlichen Kennzahlen dar, die im Zusammenhang mit der Nutzung des Kapitalstocks stehen. So entwickelten sich etwa das reale BIP oder die Nutzung der Verkehrsinfrastruktur in den letzten Jahren weitaus stärker als der öffentliche Kapitalstock (Dullien und Rietzler, 2019; Bardt et al., 2019).

Einen weiteren komparativen Ansatz stellt der Vergleich mit anderen Ländern oder Gebietskörperschaften dar. Besonders eindrücklich zeigt sich die deutsche Investitionslücke im internationalen Vergleich (vgl. Abbildung 1): Pro Einwohner investierte der deutsche Staat zwischen 2000 und 2017 jährlich preis- und kaufkraftbereinigt 845 Euro. Im EU-Vergleich lag Deutschland damit auf dem achtletzten Platz - lediglich vor den osteuropäischen Staaten Bulgarien, Lettland, Litauen, Rumänien, Slowakei, Polen und Ungarn. Diese Investitionszurückhaltung ist jedoch nicht auf einen hohen Bestand öffentlichen Kapitals vor 2000 zurückzuführen: Länder mit ähnlich hohem pro-Kopf-Kapitalstock wie Belgien, Italien oder Frankreich haben deutlich mehr investiert.'

1 In der IMF-Datenbank stehen lediglich Daten zum Nettoanlagevermögen zur Verfügung - diese haben bezüglich des Produktionspotenzials eine geringere Aussagekraft als das Bruttoanlagevermögen (Grömling et al., 2019b). 


\section{Abbildung 1}

Durchschnittliche jährliche Bruttoanlageinvestitionen und öffentliches Nettoanlagevermögen

in \% der Bevölkerung 2000, konstante internationale Dollar von 2011

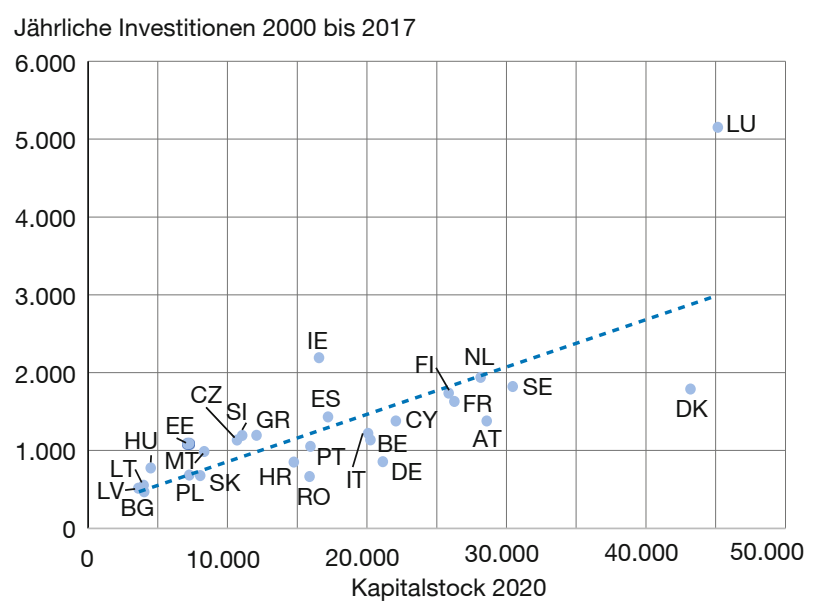

AT $=$ Österreich, BE = Belgien, BG = Bulgarien, CY = Zypern, CZ = Tschechien, $\mathrm{DE}=$ Deutschland, DK = Dänemark, EE = Estland, ES = Spanien, $\mathrm{FI}=$ Finnland, FR = Frankreich, GR = Griechenland, HR = Kroatien, HU = Ungarn, IE = Irland, IT = Italien, LT = Litauen, LU = Luxemburg, LV = Lettland, $\mathrm{MT}=$ Malta, $\mathrm{NL}=$ Niederlande, $\mathrm{PL}=$ Polen, $\mathrm{PT}=$ Portugal, $\mathrm{RO}=$ Rumänien, SE = Schweden, $\mathrm{SI}=$ Slowenien, $\mathrm{SK}=$ Slowakei.

Quellen: IMF; Eurostat; Institut der deutschen Wirtschaft.

\section{Ungenutzte fiskalische Spielräume für Investitionen}

Dabei kann auf ein Jahrzehnt hoher fiskalischer Erträge zurückgeblickt werden. Getrieben durch eine beachtliche Arbeitsmarktentwicklung - die Erwerbstätigenquote der Altersgruppe 20 bis 64 Jahre stieg in der letzten Dekade von $75 \%$ auf über $80 \%$ an - lag das nominale gesamtstaatliche Aufkommen aus Einkommen- und Vermögensteuern 2019 um rund 50\% über dem Niveau von 2011. Gleichzeitig ergab sich zwischen 2012 und 2019 eine Zinsersparnis von 204 Mrd. Euro. Zusammengenommen ergab sich bei Bund, Ländern und Gemeinden im Zeitraum 2012 bis 2019 ein fiskalischer Spielraum von rund 1,3 Billionen Euro im Vergleich zum Jahr 2011. ${ }^{2}$ Dies stellt lediglich eine Untergrenze der tatsächlichen entstandenen Spielräume dar, denn es ergaben sich weitere Einsparungen durch den Arbeitsmarktboom, die hier nicht berücksichtigt wurden: Allein der Bund sparte in den acht Jahren vor der Krise fast 50 Mrd. Euro arbeitsmarktbezogener Sozialausgaben (Bundesrechnungshof, 2020).

Diese beachtlichen fiskalischen Freiräume wurden jedoch nicht zur Schließung der Investitionslücke genutzt.

2 Ohne innerstaatliche Transfers. Der Spielraum wurde berechnet als kumulative Einnahmensteigerungen und Einsparungen bei staatlichen Zinszahlungen.

\section{Abbildung 2}

Verwendung der zusätzlichen fiskalischen Spielräume von 2012 bis 2019 im Vergleich zu 2011

in Mrd. Euro

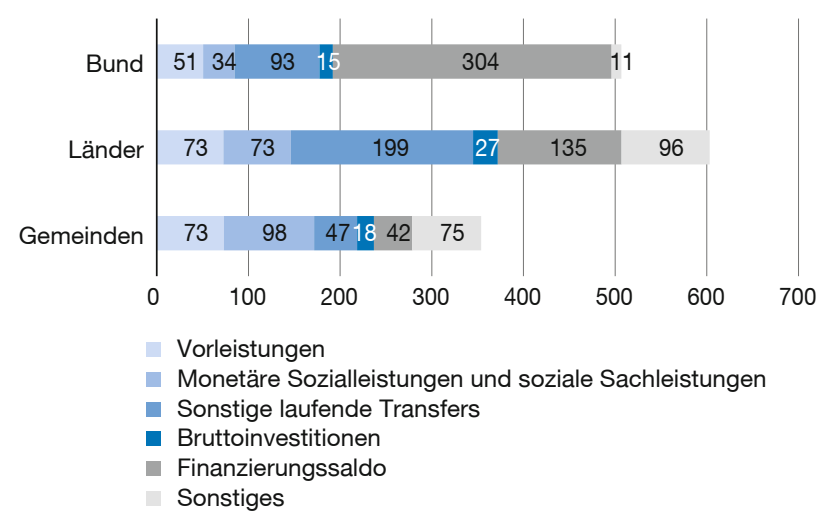

Quelle: Statistisches Bundesamt; Institut der deutschen Wirtschaft.

Im gesamten Zeitraum ergaben sich zusätzliche staatliche Investitionen von $60 \mathrm{Mrd}$. Euro, was lediglich $5 \%$ des zusätzlichen Spielraums entspricht. Dabei hätte alleine mit der Zinsersparnis $45 \%$ des von Bardt et al. (2019) ermittelten Investitionsbedarfs gedeckt werden können.

Die Verwendung der Spielräume gestaltete sich sehr unterschiedlich (vgl. Abbildung 2): Der Bund verwendete rund $60 \%$ seines zusätzlichen Spielraums (304 Mrd. von 507 Mrd. Euro) für die Reduzierung der Neuverschuldung bzw. die Schuldentilgung. Lediglich 3\% des Spielraums wurde für zusätzliche Investitionen verwendet. Den höchsten zusätzlichen Spielraum hatten die Bundesländer. Von zusammengenommen 603 Mrd. Euro investierten sie 27 Mrd. zusätzlich - rund die Hälfte ihrer Zinsersparnis. Die Gemeinden nutzten 5,2\% ihrer zusätzlichen fiskalischen Spielräume für Investitionen, $28 \%$ für monetäre Sozialleistungen und soziale Sachleistungen. Rund $60 \%$ des zusätzlichen Spielraums flossen hier in Konsumausgaben. Dabei ist zu beachten, dass sich rund die Hälfte der zusätzlichen kommunalen Spielräume aus zusätzlichen laufenden Transfers innerhalb des Staatssektors speisten.

\section{Staatlicher Investitionsboom seit 2015?}

Trotz der zuvor skizzierten Entwicklung - relativ schwache Investitionstätigkeit seit der Jahrtausendwende trotz freiwerdender fiskalischer Spielräume - setzte seit 2015 eine Steigerung staatlicher Bruttoanlageinvestitionen ein: In dem Zeitraum von 2015 bis 2019 war ein preisbereinigtes jährliches Wachstum von durchschnittlich 4,3\% zu verzeichnen. Im Vergleich zur ersten Hälfte der Dekade lagen die realen Bruttoanlageinvestitionen des Staates um $10 \%$ höher. 
Diese Steigerung entspricht aber noch nicht dem zusätzlichen Bedarf von 457 Mrd. Euro, den Bardt et al. (2019) identifizieren. 2019 stiegen die Bruttoanlageinvestitionen preisbereinigt um 3 Mrd. Euro, 2020 um 3,9 Mrd. Euro. ${ }^{3}$ Blieben die öffentlichen Investitionen in der anstehenden Dekade auf dem - vermeintlich hohen - Niveau von 2020, würden sich gegenüber 2019 zusammengenommen Mehrinvestitionen von 39 Mrd. Euro ergeben - fernab der tatsächlichen Bedarfe (vgl. Abbildung 3). Schreibt man den Trend seit dem Einsetzen des „Investitionsbooms“ 2015 linear fort, ergeben sich bis 2030 preisbereinigte Mehrinvestitionen von insgesamt 225 Mrd. Euro gegenüber 2019. Selbst wenn sich die öffentlichen Investitionssteigerungen der letzten Jahre so fortsetzten, wären sie immer noch nicht bedarfsgerecht.

\section{Investitionen im Detail}

Beim Bund schlug sich die zunehmende Dynamik in einem 2019 preisbereinigt $7 \%$ höheren Bruttoanlagevermögen als im Jahr 2014 nieder. Vor allem seit Ausbruch der CoronaKrise investiert der Bund deutlich mehr: 2020 lagen die Investitionen mit 50,3 Mrd. Euro nominal rund ein Drittel über dem Vorjahr - allerdings ein gutes Stück unter den ursprünglich veranschlagten 71,3 Mrd. Euro. Dies ist in Teilen auf nicht abgerufene Gewährleistungen und Darlehen und die Verschiebung einer Eigenkapitalerhöhung der deutschen Bahn auf 2021 zu erklären (BMF, 2021b). 2021 sollen die Bundesinvestitionen noch einmal ähnlich stark wie im Vorjahr auf rund 62 Mrd. Euro ansteigen, danach aber wieder auf 48 Mrd. Euro sinken (BMF, 2021a). Doch auch die aktuellen Erhöhungen der Bundesinvestitionen sind in Teilen der Bekämpfung von Krisenfolgen zuzurechnen und decken demnach nur partiell die strukturellen Bedarfe. Von den ursprünglich veranschlagten 71,3 Mrd. Euro für 2020 waren $45 \%$ für den Erwerb von Beteiligungen etc., Darlehen, Inanspruchnahme aus Gewährleistungen oder Zuschüsse für Investitionen im Ausland vorgesehen - lediglich 39,2 Mrd. für Baumaßnahmen, Sachinvestitionen, Investitionszuweisungen an den öffentlichen Bereich oder Zuschüsse für Investitionen im Inland (Jung und Obst, 2020).

Die Bundesländer konnten ihren Kapitalstock seit 2014 ähnlich stark wie der Bund ausbauen. Preisbereinigt lag ihr Bruttoanlagevermögen 2019 um 8\% höher als noch fünf Jahre zuvor. Dabei ist die Zunahme der Investitionstätigkeit sehr heterogen: Laut Kassenstatistik lagen die nominalen Sachinvestitionen zwischen 2015 und 2019 im Vergleich zu den vorangegangenen fünf Jahren um durchschnittlich $17 \%$ höher - bei einem Anstieg von $59 \%$ in Schleswig-Holstein an der Spitze und einem Rückgang um $20 \%$ im Saarland am unteren Ende.

3 Allerdings sind nicht alle Bestandteile des Bedarfs zu den Bruttoanlageinvestitionen zu zählen, z.B. Teile der Bildungsausgaben.

\section{Abbildung 3}

Bruttoanlageinvestitionen des Staates

in Preisen von 2019, lineare Fortschreibung des Trends 2015 bis 2020

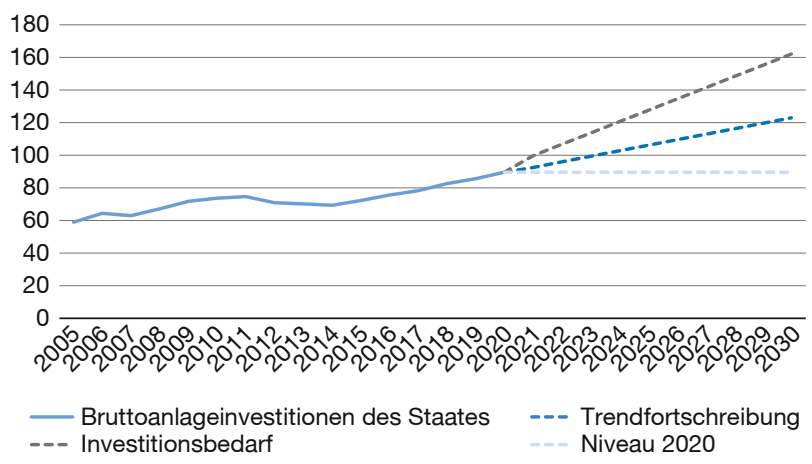

Quelle: Statistisches Bundesamt; Institut der deutschen Wirtschaft.

Bei den Gemeinden ging das Bruttoanlagevermögen zwischen 2014 und 2019 trotz nominaler Ausgabensteigerungen zurück. Dies ist vor allem auf den hohen Anteil an Bauinvestitionen der Gemeinden zurückzuführen, bei denen starke Preissteigerungen zu verzeichnen waren. Zwar sank der wahrgenommene Investitionsrückstand 2018 von 158,8 Mrd. auf 138,4 Mrd. Euro - stieg jedoch 2019 wieder auf 147 Mrd. Euro an (Krone und Scheller, 2018; 2019; 2020). Auf Gemeindeebene zeigt sich zudem eine starke Zweiteilung: In den sechs Flächenländern Niedersachsen, Mecklenburg-Vorpommern, Bayern, Schleswig-Holstein, Nordrhein-Westfalen und Baden-Württemberg lagen die nominalen Sachinvestitionen zwischen 2015 und 2019 um durchschnittlich $34 \%$ höher als in den fünf Jahren zuvor. In den anderen sieben Flächenländern gab es praktisch keinen nominalen Zuwachs. Vor allem in Westdeutschland zeigt sich ein stark negativer Zusammenhang zwischen Gemeindeverschuldung und Investitionszunahme. Wie Beznoska und Kauder (2019; 2020) zeigen, werden kommunale Investitionen insbesondere von hohen Kassenkrediten sowie Sozial- und Personalausgaben beeinträchtigt.

Neben der Analyse staatlicher Teilsektoren ist bedeutsam, in welchen Aufgabenfeldern die Investitionssteigerungen wirkten. Das gesamte staatliche Bruttoanlagevermögen lag 2019 um 3,1\% über dem des Jahres 2014. Den stärksten Zuwachs gab es bei Ausrüstungen und beim geistigen Eigentum mit einer jeweils 20\%igen Steigerung. Das Anlagevermögen bei den Wohnbauten stieg um 8,4\%. Bei Nichtwohnbauten hingegen war eine durchgehende Stagnation zu verzeichnen. Eine Ausnahme im Bereich der Nichtwohnbauten bilden die Bundesautobahnen - mit einer Steigerung von 7,4\%, bei Straßen insgesamt um 1,8\% (Statistisches Bundesamt, 2020). Dies bedeutet allerdings nicht, dass in den zurückliegenden Jahren ein bemerkenswerter Ausbau 
der Verkehrsinfrastruktur zu beobachten war. Die Länge überörtlicher Straßen liegt seit über 20 Jahren nahezu unverändert bei rund 230.000 Kilometern, die EisenbahnStreckenlänge sank um mehr als $5 \%$. Dabei wuchs das Verkehrsaufkommen stark an: Die Jahresfahrleistung auf deutschen Autobahnen stieg im gleichen Zeitraum um ein Viertel, der Bahnverkehr leistete 2019 42\% Personenkilometer und $64 \%$ Tonnenkilometer im Güterverkehr mehr als 2002 (BMVI, 2020). Gleichzeitig offenbaren Alter und Zustand der Verkehrsinfrastruktur hohe Investitionsbedarfe (Puls, 2020). Im Bildungsbereich lagen die nominalen staatlichen Bruttoanlageinvestitionen zwischen 2015 und 2019 um lediglich $0,1 \%$ über den fünf Jahren zuvor - bei Preissteigerungen von 9,1\%. ${ }^{4}$ Bedeutend stärkere Zuwächse gab es im Aufgabenfeld soziale Sicherung $(+77,4 \%)$ und der allgemeinen Verwaltungstätigkeit (+ 40,7\%).

\section{Fazit}

Insgesamt herrscht trotz bemerkenswerter Zunahme öffentlicher Investitionstätigkeit seit 2015 weiterhin ein beachtlicher Investitionsbedarf. Die positive Dynamik der letzten Jahre hat die Position Deutschlands im internationalen Vergleich nicht verbessert: 2019 investierte der deutsche Staat 1.031 Euro pro Einwohner - rund 10\% mehr als im EU-Schnitt (940 Euro), aber noch deutlich unter Frankreich (1.320 Euro). Die Steigerungen beim Bund sind in großen Teilen der Kompensation der Corona-Pandemie zuzurechnen und werden ab 2022 wieder absinken. Wichtige Aufgabengebiete wie das Bildungswesen oder die Verkehrsinfrastruktur konnten von den zusätzlichen Investitionen der letzten Jahre nicht hinreichend profitieren. Auch zwischen den Bundesländern und Gemeinden zeigen sich beachtliche Unterschiede in der Investitionsdynamik, die in Teilen auf unzureichende Mittel zurückzuführen sind. So geben laut KFW-Kommunalpanel $44 \%$ der Kommunen fehlende finanzielle Mittel als Faktor für den Investitionsrückstand an. Weitere Investitionshemmnisse sind unzureichende Kapazitäten in der Bauwirtschaft und der kommunalen Verwaltung (Krone und Scheller, 2020).

So wird mit Beginn der neuen Legislaturperiode eine strategische Antwort auf die Investitionslücke zu finden sein. Denn die anstehenden Herausforderungen im Strukturwandel verlangen insbesondere für die Dekarbonisierung nicht nur verlässliche regulatorische Rahmenbedingungen, sondern ebenso wichtige infrastrukturelle Leistungen des Staates, für die bisher beispielsweise im Sondervermögen „Energie- und Klimafonds“ durch die Bundesregierung nur erste Finanzierungen bereitgestellt wurden. Die digitale Transformation verlangt darüber hinaus eine forcierte Anstrengung,

4 Investitionen machen jedoch lediglich $7 \%$ der Bildungsausgaben aus (Beznoska et al., 2021). um die in der Pandemie offenbar gewordenen Defizite auszugleichen. All dies wird mit der bestehenden Schuldenregulierung so einfach nicht gehen, zumal die Investitionsschwäche bei den Kommunen strukturelle Verbesserungen der dortigen Finanzausstattung verlangt (Hüther, 2020). Die Investitionslücke wird nur zu schließen sein, wenn man zugleich den Reformbedarf der Schuldenbremse (Hüther und Südekum, 2020) und die Probleme im administrativen Staatsaufbau Deutschlands angeht, die sich mit der Länderhoheit für die Verwaltung und der kommunalen Selbstverwaltung in Zeiten von Krisen, aber auch grundsätzlich durch die digitale Transformation verbinden (Hüther, 2021).

\section{Literatur}

Bardt, H., S. Dullien, M. Hüther und K. Rietzler (2019), Für eine solide Finanzpolitik: Investitionen ermöglichen!, IW-Policy Paper, 10/19.

Beznoska, M. und B. Kauder (2019), Verschuldung und Investitionen der Kommunen in Deutschland, IW-Trends, 46(3), 3-19.

Beznoska, M. und B. Kauder (2020), Schieflagen der kommunalen Finanzen: Ursachen und Lösungsansätze, IW-Policy Paper, 15/20.

Beznoska, M., B. Kauder, und T. Obst (2021), Invetitionen, Humankapital und Wachstumswirkungen öffentlicher Ausgaben, IW-Policy Paper, $2 / 21$.

BMF (2021a), BMF-Monatsbericht Februar 2021, Bundesministerium der Finanzen.

BMF (2021b), BMF-Monatsbericht Januar 2021, Bundesministerium der Finanzen.

BMVI (2020), Verkehr in Zahlen 2020/2021, Bundesministerium für Verkehr und digitale Infrastruktur.

Bundesrechnungshof (2020), Bericht an den Haushaltsausschuss des Deutschen Bundestages nach $\S 88$ Abs. 2 BHO: Feststellungen zur finanzwirtschaftlichen Entwicklung des Bundes - ein weiter Weg aus der Krise.

Dullien, S. und K. Rietzler (2019), Betrachtung des Bruttokapitalstocks mit massiven Schwierigkeiten behaftet - eine Replik, Wirtschaftsdienst, 99(4), 286-291.

Feld, L. P., W. H. Reuter und M. Yeter (2020), Öffentliche Investitionen: Die Schuldenbremse ist nicht das Problem, Freiburger Diskussionspapiere zur Ordnungsökonomik, No. 20/01.

Grömling, M., M. Hüther und M. Jung (2019a), Basis für evidenzbasierte Politik: aussagekräftiges Bruttoanlagevermögen - eine Erwiderung, Wirtschaftsdienst, 99(4), 291-294.

Grömling, M., M. Hüther und M. Jung (2019b), Verzehrt Deutschland seinen staatlichen Kapitalstock?, Wirtschaftsdienst, 99(1), 25-31.

Hüther, M. (2020), Zeit für Wachstumspolitik, Wirtschaftsdienst, 100(3), 165-169.

Hüther, M. (2021), Der Staat als Retter und die Mühen der administrativen Ebene, Makronom, https://makronom.de/der-staat-als-retter-unddie-muehen-der-administrativen-ebene-38510 (3. März 2021).

Hüther, M. und J. Südekum (2020), Die Schuldenbremse nach der Corona-Krise, Wirtschaftsdienst, 100(10), 746-752.

Jung, M. und T. Obst (2020), Kein Wumms bei öffentlichen Investitionen, IW-Kurzbericht, 113/2020.

Krone, E. und H. Scheller (2018), KfW-Kommunalpanel 2018, KfW Bankengruppe.

Krone, E. und H. Scheller (2019), KfW-Kommunalpanel 2019, KfW Bankengruppe.

Krone, E. und H. Scheller (2020), KfW-Kommunalpanel 2020, KfW Bankengruppe.

Peacock, A. und J. Wiseman (1961), The Growth of Public Expenditure in the United Kingdom, Princeton University Press.

Puls, T. (2020), Jenseits des Geldes: Was behindert den Infrastrukturausbau in Deutschland, IW-Report, 37/20.

Statistisches Bundesamt (2020), Volkswirtschaftliche Gesamtrechnungen: Arbeitsunterlage Investitionen: 3. Vierteljahr 2020. 